\title{
New Insights Into Functions and Possible Applications of Clostridium difficile CRISPR-Cas System
}

\author{
Anna Maikova ${ }^{1,2,3,4}$, Konstantin Severinov ${ }^{1,4,5}$ and Olga Soutourina ${ }^{3,6 *}$ \\ ${ }^{1}$ Center for Life Sciences, Skolkovo Institute of Science and Technology, Moscow, Russia, ${ }^{2}$ Université Paris Diderot, \\ Sorbonne Paris Cité, Paris, France, ${ }^{3}$ Microbiology, Institute for Integrative Biology of the Cell, Commissariat à l'Energie \\ Atomique et aux Energies Alternatives, Centre National de la Recherche Scientifique, Université Paris-Sud, Université \\ Paris-Saclay, Gif-sur-Yvette, France, ${ }^{4}$ Peter the Great St. Petersburg Polytechnic University, Saint Petersburg, Russia, \\ ${ }^{5}$ Waksman Institute for Microbiology, Rutgers, The State University of New Jersey, Piscataway, NJ, United States, ${ }^{6}$ Institut \\ Pasteur, Paris, France
}

\section{OPEN ACCESS}

Edited by:

Meina Neumann-Schaal, Deutsche Sammlung von Mikroorganismen und Zellkulturen

(DSMZ), Germany

Reviewed by: Konstantinos Papadimitriou, Agricultural University of Athens,

Greece

Meera Unnikrishnan University of Warwick, United Kingdom

*Correspondence: Olga Soutourina olga.soutourina@i2bc.paris-saclay.fr

Specialty section: This article was submitted to Infectious Diseases,

a section of the journal Frontiers in Microbiology

Received: 30 May 2018 Accepted: 12 July 2018 Published: 31 July 2018

Citation:

Maikova A, Severinov K and Soutourina O (2018) New Insights Into Functions and Possible Applications of Clostridium difficile CRISPR-Cas System. Front. Microbiol. 9:1740. doi: 10.3389/fmicb.2018.01740
Over the last decades the enteric bacterium Clostridium difficile (novel name Clostridioides difficile) - has emerged as an important human nosocomial pathogen. It is a leading cause of hospital-acquired diarrhea and represents a major challenge for healthcare providers. Many aspects of $C$. difficile pathogenesis and its evolution remain poorly understood. Efficient defense systems against phages and other genetic elements could have contributed to the success of this enteropathogen in the phagerich gut communities. Recent studies demonstrated the presence of an active CRISPR (clustered regularly interspaced short palindromic repeats)-Cas (CRISPR-associated) subtype I-B system in C. difficile. In this mini-review, we will discuss the recent advances in characterization of original features of the $C$. difficile CRISPR-Cas system in laboratory and clinical strains, as well as interesting perspectives for our understanding of this defense system function and regulation in this important enteropathogen. This knowledge will pave the way for the development of promising biotechnological and therapeutic tools in the future. Possible applications for the $C$. difficile strain monitoring and genotyping, as well as for CRISPR-based genome editing and antimicrobials are also discussed.

Keywords: CRISPR, C. difficile, I-B subtype CRISPR-Cas system, prophage, CRISPR regulation, stress, antimicrobials, genome editing

\section{CRISPR-Cas SYSTEMS: GENERAL FUNCTIONAL ASPECTS AND CLASSIFICATION}

The CRISPR (clustered regularly interspaced short palindromic repeats)-Cas (CRISPR-associated) systems are adaptive immune systems protecting prokaryotes against phages and other mobile genetic elements (Sorek et al., 2013). These defensive systems are found in almost all sequenced archaeal and in about half of bacterial genomes (Grissa et al., 2007). CRISPR-Cas systems are composed of CRISPR arrays and cas operons. CRISPR arrays in turn consist of short direct repeats (20-40 bp) separated by variable spacers. Some spacers are complementary to mobile genetic elements sequences (Shmakov et al., 2017). CRISPR arrays also contain leader regions carrying promoters from which their transcription initiates. 
CRISPR-based defensive functions include two major processes: immunity (interference) and immunization (adaptation) (for general review, see Marraffini, 2015). CRISPR interference itself can be divided into two phases: the biogenesis of CRISPR RNAs and the targeting phase. At the first phase a CRISPR array is transcribed into a long RNA transcript (precrRNA), which is processed into small CRISPR RNAs (crRNAs), each consisting of one spacer and flanking repeat sequences. Individual crRNAs bind to Cas proteins forming a nucleoprotein effector complex, which is necessary for the second, targeting phase. The crRNAs serve as guides for recognizing nucleic acids by complementary base pairing. In this way, crRNAs direct recognition and, ultimately, cleavage of genetic elements by the Cas nucleases (Garneau et al., 2010). Spacers are incorporated into CRISPR arrays in the process of adaptation (Jackson et al., 2017). Cas 1 and Cas 2 proteins, found in almost all investigated CRISPR-Cas systems, are essential for this process (Koonin et al., 2017). A very important aspect of CRISPR-based immunity is the ability to distinguish host DNA from the foreign one. Protospacer-adjacent motifs (PAMs) are short sequences situated on the $3^{\prime}$ or $5^{\prime}$ end of the protospacer (foreign DNA region corresponding to a CRISPR spacer) and required for protospacer recognition. PAMs are absent from CRISPR arrays, which prevents autoimmunity (Sorek et al., 2013). PAMs are essential during spacer selection at the adaptation stage, which ensures that acquired spacers are functional in interference. Previous studies in type I CRISPR-Cas systems identified the sequence requirements for the CRISPR targeting that includes a perfect match between the $5^{\prime}$ end of the spacer and the protospacer within up to a 10-nt "seed" sequence (Semenova et al., 2011; Wiedenheft et al., 2011; Maier et al., 2013a).

CRISPR-Cas systems are highly diverse. This is reflected in both CRISPR array architectures and cas genes composition (Takeuchi et al., 2012). The variability of cas gene sets has formed the basis of CRISPR-Cas systems classification (Makarova et al., 2011). All investigated CRISPR-Cas systems are divided into two classes, characterized by the composition of cas genes involved in interference module (Koonin et al., 2017). These classes in turn are divided into six types and 33 subtypes (see Table $\mathbf{1}$ for examples). The Class 1 comprises the most abundant and diverse type I and type III CRISPR-Cas systems as well as rare type IV. These types of CRISPR-Cas systems are found in both archaeal and bacterial genomes. Effector complexes of the type I and type III include Cas5, Cas7, Cas8 (in type I), and Cas10 (in type III) proteins (Koonin et al., 2017). For crRNA processing Cas6 family proteins are necessary in these CRISPR-Cas systems (Charpentier et al., 2015). Type I systems are also characterized by the presence of Cas3 proteins responsible for degradation of DNA recognized by effector complexes (Brouns et al., 2008). The Class 2 includes type II, type V and type VI CRISPR-Cas systems. These systems possess effector modules consisting of only one multi-domain protein. The most characterized is the type II Cas9 protein widely used in genome editing (Wang et al., 2016).

The type I CRISPR-Cas systems are highly diverse and subdivided into seven subtypes (I-A, I-B, I-C, I-U, I-D, I-E, I-F) (Makarova et al., 2015). The subtypes I-C, I-D, I-E, I-F are encoded by a single operon in CRISPR loci, whereas subtype I-A and I-B are often encoded by several operons. I-C, I-E, and I-F subtypes are mostly present in bacteria, while I-A, I-B, and I-D are common in archaea (Makarova et al., 2011) (Table 1). The subtype I-B, characterized by a specific Cas8b protein, is present in methanogenic and halophilic archaea and in clostridia. Studies of the I-B CRISPRCas systems in haloarchaea showed some interesting features such as multiple PAMs and 9-nucleotide non-contiguous seed region (Maier et al., 2015). Although the subtype I-B was found in clostridial species it has not been well studied there yet. It is suggested that I-B CRISPR-Cas system possibly had been acquired by clostridia from archaea via horizontal gene transfer and afterward evolved independently (Peng et al., 2014). Other CRISPR-Cas systems subtypes, including I-A, I-C, III-A, III-B, and II-C, are also present in some clostridial species (Table 1).

\section{CHARACTERIZATION OF Clostridium difficile CRISPR-Cas SYSTEM}

Clostridium difficile (novel name Clostridioides difficile) is an anaerobic spore-forming bacterium, one of the major clostridial pathogens and the major cause of nosocomial infections associated with antibiotic therapy (Abt et al., 2016). During its infection cycle, this enteropathogen must cope with the presence of foreign DNA elements, including bacteriophages, in the crowded environment of the gut, and is thus expected to rely on efficient defense systems such as CRISPR-Cas to control genetic exchanges favored in its complex niche.

The first evidence suggesting the presence of active CRISPRCas system in C. difficile was obtained during deep-sequencing of regulatory RNAs in C. difficile (Soutourina et al., 2013). This study revealed abundant and diverse crRNAs. Active expression and processing of CRISPR loci was detected in this and a subsequent study (Soutourina et al., 2013; Boudry et al., 2015).

Bioinformatics analysis of more than 200 C. difficile genomes (Hargreaves et al., 2014; Andersen et al., 2016) demonstrated that $C$. difficile CRISPR-Cas system belongs to I-B subtype (Koonin et al., 2017). C. difficile CRISPR-Cas system possesses several original features (Figure 1). CRISPR-Cas system of this enteropathogen is characterized by an unusual large set of CRISPR arrays. For example, reference 630 and hypervirulent R20291 C. difficile strains contain 12 and 9 CRISPR arrays, respectively (Soutourina et al., 2013; Boudry et al., 2015). These CRISPR arrays are orientated in the direction of chromosome replication, as observed for highly expressed bacterial genes and presumably ensuring their optimal transcription (Arakawa and Tomita, 2007; Boudry et al., 2015). On average, known C. difficile genomes contain 8.5 arrays (Andersen et al., 2016). In most sequenced $C$. difficile strains several CRISPR arrays are located in prophages (Hargreaves et al., 2014; Boudry et al., 2015). The crRNAs originating from arrays located in prophages were found to be the most expressed in 630 and R20291 strains. Prophage localization of actively expressed CRISPR arrays may play a role in preventing infection by related competing phages by targeting their DNA (Sorek et al., 2008). 
Another unusual feature of $C$. difficile CRISPR-Cas system is the presence of two or three (in 027 ribotype strains) cas gene sets in the majority of sequenced strains (Boudry et al., 2015) (Figure 1). The full cas operon encodes all necessary genes for CRISPR interference (cas6, cas8b, cas7, cas5, cas3) as well as cas1, cas2, cas4 genes essential for spacer acquisition (Amitai and Sorek, 2016; Kieper et al., 2018; Lee et al., 2018). The additional cas operons lack the adaptation module. While the complete cas gene operons were found in $\sim 90 \%$ of sequenced C. difficile strains, the additional partial cas gene sets are present in almost all strains (Boudry et al., 2015). Thus, some C. difficile strains could have lost the ability to adapt to new genetic elements through their CRISPR-Cas systems. The cas operon occurrence correlates with evolutionary relationships of $C$. difficile strains reflecting their epidemiological context and, possibly, the intensity of interactions with foreign DNA elements (Boudry et al., 2015). When present, complete cas gene operons are usually associated with longest CRISPR arrays, which is indicative of active new spacer acquisition (or slower spacer loss) and hints to an existence of some still unknown in cis mechanisms responsible for different dynamics of cas proximal arrays. The conservation of CRISPR array structure and sequences of all CRISPR repeats in C. difficile

TABLE 1 | Main CRISPR-Cas systems subtypes and examples of system-harboring microorganisms and clostridial species.

\begin{tabular}{|c|c|c|c|c|}
\hline Class & Subtype & cas operon composition & Example & Examples of clostridial species and strains \\
\hline \multirow[t]{11}{*}{ Class 1} & I-A & $\begin{array}{l}\text { cas6, cas11(csa5), cas7, cas5, cas8a1, cas3', } \\
\text { cas3", cas2, cas4, cas1, cas4 }\end{array}$ & $\begin{array}{l}\text { Listeria monocytogenes L99 (Sesto } \\
\text { et al., 2014) }\end{array}$ & $\begin{array}{c}\text { C. stercorarium subsp. stercorarium DSM } 8532 \\
\text { (Poehlein et al., 2013); C. tetani ATCC } 9441 \\
\text { (Cohen et al., 2017) }\end{array}$ \\
\hline & I-B & $\begin{array}{l}\text { cas6, cas8b1, cas7, cas5, cas3, cas4, cas1, } \\
\text { cas2 }\end{array}$ & $\begin{array}{l}\text { Haloferax volcanii H119 (Maier et al., } \\
\text { 2013b) }\end{array}$ & $\begin{array}{l}\text { C. difficile 630, C. difficile R20291 (Boudry } \\
\text { et al., 2015); C. pasteurianum BC1 (Pyne et al., } \\
\text { 2016); C. acetobutylicum GXAS18-1 (Peng } \\
\text { et al., 2014); C. tetani ATCC } 9441 \text { (Cohen et al., } \\
\text { 2017) }\end{array}$ \\
\hline & $\mathrm{I}-\mathrm{C}$ & cas3, cas5, cas8c, cas7, cas4, cas1, cas2 & $\begin{array}{l}\text { Desulfovibrio vulgaris str. } \\
\text { Hildenborough (Hochstrasser et al., } \\
\text { 2016) }\end{array}$ & C. cellulolyticum H10 (Brown et al., 2014) \\
\hline & $\mathrm{I}-\mathrm{U}$ & $\begin{array}{l}\text { cas3, cas8u2, cas7, cas5-cas6, cas4-cas1, } \\
\text { cas2 }\end{array}$ & $\begin{array}{l}\text { Geobacter sulfurreducens (Koonin } \\
\text { et al., 2017) }\end{array}$ & - \\
\hline & I-D & $\begin{array}{l}\text { cas3', cas3", cas10d, cas7(csc2), cas5(csc1), } \\
\text { cas6, cas4, cas1, cas2 }\end{array}$ & $\begin{array}{l}\text { Cyanothece sp. } 8802 \text { (Koonin et al., } \\
\text { 2017) }\end{array}$ & - \\
\hline & I-E & $\begin{array}{l}\text { cas3, cas8e(cse1), cas11(cse2), cas7, cas5, } \\
\text { cas6, cas1, cas2 }\end{array}$ & $\begin{array}{l}\text { Escherichia coli K12 (Koonin et al., } \\
\text { 2017) }\end{array}$ & - \\
\hline & $\mathrm{I}-\mathrm{F}$ & $\begin{array}{l}\text { cas1, cas2-cas3, cas8f(csy1), cas5(csy2), } \\
\text { cas7(csy3), cas6f }\end{array}$ & $\begin{array}{l}\text { Pseudomonas aeruginosa PA14 } \\
\text { (Wiedenheft et al., 2011) }\end{array}$ & - \\
\hline & $\| I I-A$ & $\begin{array}{l}\text { cas6, cas10, cas11(csm2), cas7(csm3), } \\
\operatorname{cas5}(\mathrm{csm} 4), \operatorname{cas} 7(\mathrm{csm} 5), \operatorname{csm} 6, \operatorname{cas} 1, \operatorname{cas} 2\end{array}$ & $\begin{array}{l}\text { Staphylococcus epidermidis (Koonin } \\
\text { et al., 2017) }\end{array}$ & C. tetani ATCC 453 (Cohen et al., 2017) \\
\hline & III-B & $\begin{array}{l}\operatorname{cas} 7(\mathrm{cmr1}), \operatorname{cas} 10, \operatorname{cas5}(\mathrm{cmr} 3), \operatorname{cas} 7(\mathrm{cmr} 4), \\
\operatorname{cas11}(\mathrm{cmr} 5), \operatorname{cas} 6, \operatorname{cas7}(\mathrm{cmr} 6)\end{array}$ & $\begin{array}{l}\text { Pyrococcus furiosus (Koonin et al., } \\
\text { 2017) }\end{array}$ & $\begin{array}{l}\text { C. botulinum ATCC } 3502 \text { (Negahdaripour et al., } \\
\text { 2017) }\end{array}$ \\
\hline & III-C & $\begin{array}{l}\text { cas7(cmr1), cas7(cmr6), cas10, cas7(cmr4), } \\
\text { cas11(cmr5), cas5(cmr3) }\end{array}$ & $\begin{array}{l}\text { Methanothermobacter } \\
\text { thermautotrophicus (Koonin et al., } \\
\text { 2017) }\end{array}$ & - \\
\hline & $\| I I-D$ & $\begin{array}{l}\operatorname{cas10}, \operatorname{cas} 7(\mathrm{csm} 3), \operatorname{cas} 5(\operatorname{cs} \times 10), \operatorname{cas} 11(\mathrm{csm} 2) \\
\operatorname{cas} 7(\mathrm{csm} 7), \operatorname{cas} 7(\mathrm{csm} 5), \text { all1473, cas7(csm5) }\end{array}$ & $\begin{array}{l}\text { Synechocystis sp. } 6803 \text { (Makarova } \\
\text { et al., 2015) }\end{array}$ & - \\
\hline \multirow[t]{8}{*}{ Class 2} & $\|-A$ & cas9, cas1, cas2, csn2 & $\begin{array}{l}\text { Enterococcus faecalis OG1RF } \\
\text { (Bourgogne et al., 2008) }\end{array}$ & - \\
\hline & II-B & cas9, cas1, cas2, cas4 & $\begin{array}{l}\text { Legionella pneumophila str. Paris } \\
\text { (Koonin et al., 2017) }\end{array}$ & - \\
\hline & $\|-C$ & cas9, cas1, cas2 & $\begin{array}{l}\text { Neisseria lactamica 020-06 (Koonin } \\
\text { et al., 2017) }\end{array}$ & C. perfringens JGS1495 (Pearson et al., 2015) \\
\hline & $V-A$ & cas12a(cpf1), cas4, cas1, cas2 & $\begin{array}{l}\text { Francisella cf. novicida Fx1 (Koonin } \\
\text { et al., 2017) }\end{array}$ & - \\
\hline & V-B & cas12b(c2c1), cas4, cas1, cas2 & $\begin{array}{l}\text { Alicyclobacillus acidoterrestris (Koonin } \\
\text { et al., 2017) }\end{array}$ & - \\
\hline & $\mathrm{V}-\mathrm{C}$ & cas1, cas12c(c2c3) & Oleiphilus sp. (Koonin et al., 2017) & - \\
\hline & V-D & cas1, cas12d(casY) & $\begin{array}{l}\text { Bacterium CG09_39_24 (Koonin et al., } \\
\text { 2017) }\end{array}$ & - \\
\hline & $V-E$ & cas12e(casX), cas4, cas1, cas2 & $\begin{array}{l}\text { Deltaproteobacteria bacterium (Koonin } \\
\text { et al., 2017) }\end{array}$ & - \\
\hline
\end{tabular}




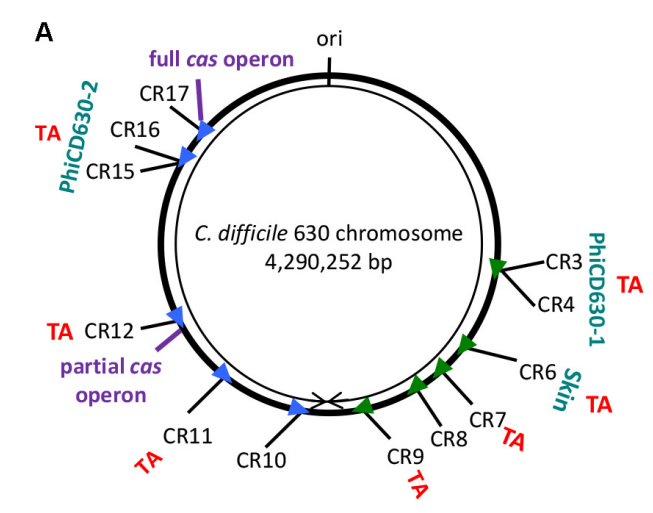

i

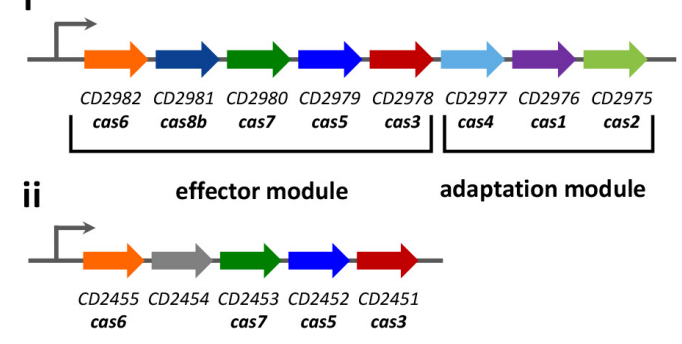

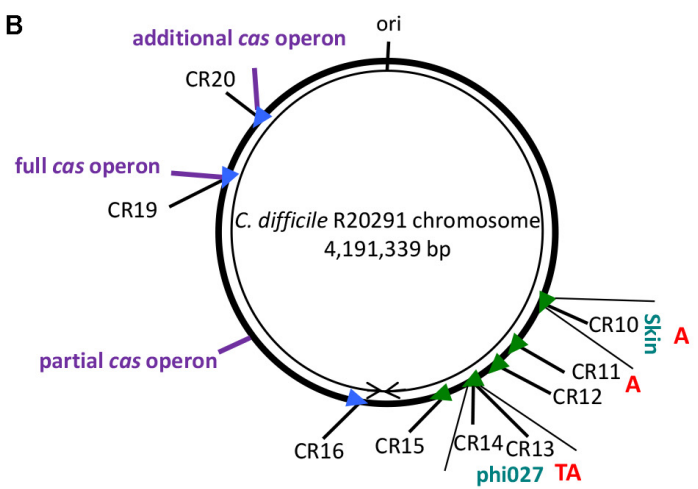

i

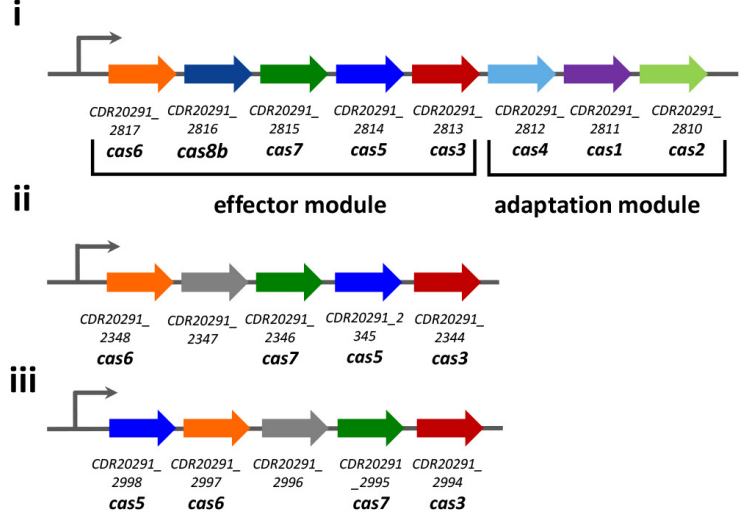

FIGURE 1 | Schematic view of the chromosomal location of CRISPR arrays and the organization of the cas operons in C. difficile strains 630 (A) and R20291 (B). CRISPR arrays (CR) are numbered according to the CRISPRdb database (Grissa et al., 2007). Arrowheads signify arrays' position and transcriptional orientation. The locations of associated cas operons, prophage regions, toxin-antitoxin pairs (TA) or only antitoxins (A) and replication origin (ori) are indicated. The organization of the cas operons in strain 630 (left) and R20291 (right) are indicated with roman numerals, where i - full operons; ii - partial operons, iii - an additional operon. Functional modules are marked with braces. The same color was used for homologous cas genes (Boudry et al., 2015).

genomes suggests that CRISPR arrays located far from cas operons use the same set of Cas proteins for their function.

An interesting evolutionary aspect of $C$. difficile CRISPR-Cas system has been recently reported (Maikova et al., 2018). Analysis of about 2,500 C. difficile genomes revealed co-localization of type I toxin-antitoxin (TA) modules with CRISPR arrays (Figure 1). TA - CRISPR array co-localization has never been reported for other bacteria and its significance remains unclear. CRISPRarrays localized in prophage regions are in particular prone to be associated with type I TA modules, which may contribute to the stabilization of these chromosomal regions by the mechanism similar to plasmid maintenance through post-segregation killing.

The function of CRISPR-Cas system is to provide defense against viruses and other mobile genetic elements. Recent bioinformatics analysis of $C$. difficile CRISPR spacers matching known sequences showed that most of them target clostridial phages and prophage regions (Hargreaves et al., 2014; Boudry et al., 2015). This suggests that this entheropathogen actively interacts with phages, and that CRISPR-Cas actively modulates this interaction. Identification of protospacers allowed to deduce PAM sequences. While 3-nucleotide $5^{\prime}$-motifs CCA or CCT were most common, alternative sequences CCC, CCG, and TCA were also frequently found. Multiple PAMs were also observed in other type I-B systems (Shah et al., 2013). Conjugation efficiency experiments with plasmid vectors containing CCA and CCT PAMs and protospacers corresponding to the first spacers from actively expressed $C$. difficile 630 CRISPR arrays showed active CRISPR interference in $C$. difficile cells thus validating bioinformatically predicted PAMs and showing that C. difficile CRISPR-Cas system is naturally capable of defensive function (Boudry et al., 2015). Phage infection assays in 630 and R20291 strains revealed the correlation between the presence of CRISPR spacer-targeting phage sequences and phage susceptibility. Experiments using a heterologous E. coli host system showed that both cas operons of $C$. difficile 630 strain are capable of interference.

\section{REGULATION AND POTENTIAL ALTERNATIVE FUNCTIONS OF C. difficile CRISPR-Cas SYSTEM}

During its infection cycle $C$. difficile faces with different stress conditions and changing environments inside the host. To 
survive in phage-rich gut community while relying on the CRISPR-Cas defense, $C$. difficile needs to regulate CRISPRCas expression in response to different environmental signals. A study by Boudry et al. (2015) revealed that all the CRISPR arrays and cas genes are expressed under standard laboratory conditions. The level of CRISPR-Cas expression could be modulated by specific regulatory mechanisms.

Bacterial pathogens often form biofilms, which help them resist different threats inside the host. It was shown that C. difficile actively forms biofilms (Dapa et al., 2013; Nale et al., 2016; Soavelomandroso et al., 2017) during its infection cycle. Biofilm conditions are characterized by high cell densities, which increase the possibility of horizontal gene transfer (Babic et al., 2011; Abedon, 2012). Quorum sensing is one of bacterial mechanisms that regulates gene expression depending on the density of the population (Miller and Bassler, 2001). Recent studies showed that cas gene expression is induced by quorum sensing signals in Serratia sp. (I-E, I-F, and III-A subtypes) (Patterson et al., 2016) and Pseudomonas aeruginosa (I-F subtype) (Høyland-Kroghsbo et al., 2016). Moreover, CRISPR-Cas systems may play a role in biofilm formation and colonization of the host. For instance, CRISPR-Cas (II-A subtype) harboring Enterococcus faecalis strain has increased biofilm formation (Bourgogne et al., 2008). Furthermore, CRISPR-Cas-mediated gene regulation of the ability to swarm and form biofilms was revealed in $P$. aeruginosa (Zegans et al., 2009). In C. difficile strain 630 , a recent study revealed up to 20 -fold induction of cas genes expression in biofilms (Maikova et al., 2018), suggesting the regulation of $C$. difficile CRISPR-Cas system activity by biofilmrelated factors. During infection, the complex interactions with different microbiota members within gut communities should be considered. More studies are thus needed to assess the possible link between biofilm-related signals and the regulation of CRISPR-Cas expression during the C. difficile infection cycle.

The obvious stress to induce CRISPR-Cas system is phage infection. At the earliest stages of attachment to the cell surface, it is often accompanied by the envelope stress (Ratner et al., 2015). The induction of the CRISPR-Cas system expression in response to this type of stress was found in different bacteria (Westra et al., 2014). Bacterial pathogens and commensals always combat with the host's immune response, which results in a wide range of stressful effects. Several studies reported the changes of cas gene transcription in Desulfovibrio vulgaris (Mukhopadhyay et al., 2007), Streptococcus sanguinis (Rodriguez et al., 2011), Pasteurella multocida (Melnikow et al., 2008), and Lactobacillus rhamnosus (Koskenniemi et al., 2011) in response to different stresses such as changes in growth rate, bile, oxidative, nitrosative stresses and exposure to antibiotics. Virulence is a specific response of pathogens to different stress factors inside the host (Louwen et al., 2014). The regulation of CRISPR-Cas systems during the infection cycle may indicate an important role of these systems in pathogens. Recently, a role of an alternative SigB factor in stress response was investigated in C. difficile (Kint et al., 2017). Interestingly, SigB-dependent promoters were found upstream of both cas operons in C. difficile strain 630 (Maikova et al., 2018) and fivefold decrease in expression levels of both cas operons was observed in $\operatorname{sig} B$ mutant strain. This suggests regulation of
C. difficile CRISPR-Cas system via stress-related signals and a potential role of this system in the survival of $C$. difficile inside the host.

Besides the adaptive immunity, multiple alternative functions of CRISPR-Cas systems have been revealed (Louwen et al., 2014; Westra et al., 2014). These functions occur through targeting bacterium' own genes by partially or fully matching crRNAs. For instance, in Listeria monocytogenes a specific long type I-A CRISPR array transcript $r l i B$ processed by polynucleotide phosphorylase (PNPase) controls the expression of the feoAB genes important for virulence (Mandin et al., 2007; Sesto et al., 2014). An rliB mutant colonizes its host more effectively than the wild type strain. Bioinformatics analysis of $C$. difficile CRISPR spacers showed that all investigated strains carry genometargeting spacers (Boudry et al., 2015). It may thus be speculated that $C$. difficile CRISPR-Cas system might also have functions in the regulation of the endogenous gene expression. The possible role of CRISPR-Cas systems in genome evolution via selftargeting is actively discussed (Westra et al., 2014).

\section{POTENTIAL APPLICATIONS OF C. difficile CRISPR-Cas SYSTEM}

During the last decade, discoveries in the CRISPR field led to rapid development of revolutionary biotechnological applications especially in genome editing by CRISPR-Cas9 technology (Hsu et al., 2014). Different CRISPR-based tools have proved to be effective both in prokaryotes and eukaryotes (Hsu et al., 2014; Barrangou and Horvath, 2017).

Since spacers are acquired in an orderly manner, with more recently acquired spacer being closer to the leader sequence (Barrangou et al., 2007; Nuñez et al., 2015) the order of spacers within an array reflects phage invasions in different populations of the same bacterial species. This feature can reveal phylogenetic relations between strains and can be used in genotyping techniques (Louwen et al., 2014; Andersen et al., 2016). Such "CRISPR-typing" has been already applied for outbreak tracking of Yersinia pestis (Cui et al., 2008; Barros et al., 2014) and Salmonella enterica (Timme et al., 2013; Pettengill et al., 2014). Moreover, CRISPR typing is capable to reveal antibiotic-resistant phenotypes (Palmer and Gilmore, 2010) or prophages (Nozawa et al., 2011). These correlations can be explained by the influence of active CRISPR-Cas systems on the horizontal gene transfer, which plays important role in the acquisition of new genes and operons, essential for bacterial pathogenesis and adaptation (Louwen et al., 2014). CRISPRtyping approach based on spacer content and polymorphism can be successfully applied to $C$. difficile with correlation between CRISPR-groups and toxin groups (Andersen et al., 2016).

CRISPR-Cas systems can be applied for development of new antimicrobials based on the self-targeting (Bikard et al., 2012). The general strategy is the use of phage particles and phagemids as vectors to deliver auto-targeting CRISPR-Cas components inside a pathogenic cell (Bikard and Barrangou, 2017). Many pathogens possess endogenous active CRISPR-Cas systems, which can be repurposed for self-targeting. Since $C$. difficile 
contains a naturally active CRISPR-Cas system, such a strategy could be promising for control and even treatment of $C$. difficile infection (CDI), in the context of recent worldwide emergence of antibiotic-resistant $C$. difficile strains (Banawas, 2018). Phage therapy of CDI has proved to be another promising alternative, but it faces some difficulties including lack of appropriate phages (Hargreaves and Clokie, 2014; Sekulovic et al., 2014). The presence of active CRISPR-Cas system should effectively prevent infection by at least some phages complicating matters further.

The most popular biotechnological application of CRISPRCas systems is genome editing (Barrangou and Horvath, 2017). In prokaryotes, the most interesting is the application of endogenous CRISPR-Cas systems since it requires the introduction of less additional components for the editing process. Several works showing the applications of endogenous I-B subtype systems for genome editing were recently published. The first one, by Pyne et al. (2016) describes this approach in Clostridium pasteurianum. In this study, a plasmid vector containing an artificial CRISPR array with a protospacer targeting the gene of interest and arms for homologous recombination was used to delete the cpaAIR gene encoding a restriction enzyme (Pyne et al., 2016). This approach allows fast and markless deletion or modification of the genes of interest in bacteria. Later, other studies confirmed the efficiency of this method in other I-B subtype-carrying organisms: archaeon Haloarcula hispanica (Cheng et al., 2017) and butanol producing Clostridium tyrobutyricum (Zhang et al., 2018). Another study revealed that Haloferax volcanii CRISPR-Cas system with deletions of cas3 and cas6 genes can be used for programmable repression of genes in this archaeon (Stachler and Marchfelder, 2016). Many efficient approaches for $C$. difficile genome manipulation exist to date. ClosTron is a method based on altered type II intron, which is able to insert in almost every region of the chromosome (Kuehne et al., 2011). Another method is CodA allele exchange

\section{REFERENCES}

Abedon, S. T. (2012). Spatial vulnerability: bacterial arrangements, microcolonies, and biofilms as responses to low rather than high phage densities. Viruses 4, 663-687. doi: 10.3390/v4050663

Abt, M. C., McKenney, P. T., and Pamer, E. G. (2016). Clostridium difficile colitis: pathogenesis and host defence. Nat. Rev. Microbiol. 14, 609-620. doi: 10.1038/ nrmicro.2016.108

Amitai, G., and Sorek, R. (2016). CRISPR-Cas adaptation: insights into the mechanism of action. Nat. Rev. Microbiol. 14, 67-76. doi: 10.1038/nrmicro. 2015.14

Andersen, J. M., Shoup, M., Robinson, C., Britton, R., Olsen, K. E. P., and Barrangou, R. (2016). CRISPR Diversity and microevolution in Clostridium difficile. Genome Biol. Evol. 8, 2841-2855. doi: 10.1093/gbe/ev w203

Arakawa, K., and Tomita, M. (2007). Selection effects on the positioning of genes and gene structures from the interplay of replication and transcription in bacterial genomes. Evol. Bioinform. Online 3, 279-286. doi: 10.1177/ 117693430700300005

Babic, A., Berkmen, M. B., Lee, C. A., and Grossman, A. D. (2011). Efficient gene transfer in bacterial cell chains. mBio 2:e0027-11. doi: 10.1128/mBio.00027-11

Banawas, S. S. (2018). Clostridium difficile infections: a global overview of drug sensitivity and resistance mechanisms. Biomed Res. Int. 2018:8414257. doi: $10.1155 / 2018 / 8414257$ technique based on semi-suicidal vector carrying the $E$. coli $\operatorname{cod} A$ gene as a counter-selectable marker (Cartman et al., 2012). Successful application of CRISPR-Cas9 (McAllister et al., 2017; Wang et al., 2018) and Cpf1 (Hong et al., 2018) systems for genome editing in $C$. difficile was recently reported and may further extend our ability to manipulate the genome of this pathogen.

Despite the recent insights, many aspects of $C$. difficile CRISPR-Cas system remain to be characterized. We hope that future studies will shed new light on the secrets of $C$. difficile success within host environments relying on effective defense systems and will lead to promising medical and biotechnological applications.

\section{AUTHOR CONTRIBUTIONS}

AM wrote the draft of the paper. OS and KS designed the project and performed critical revisions of the manuscript.

\section{FUNDING}

This work was supported by grants from the Paris Diderot University, Agence Nationale de la Recherche ("CloSTARn," ANR-13-JSV3-0005-01 to OS), the Institut Universitaire de France (to OS), the Pasteur-Weizmann Council, the University Paris-Sud, the Institute for Integrative Biology of the Cell, Vernadski fellowship to AM and the Skoltech Biomedical Initiative grant (SBI RF-0000000136) to KS.

\section{ACKNOWLEDGMENTS}

We are grateful to E. Semenova for helpful discussions.

Barrangou, R., Fremaux, C., Deveau, H., Richards, M., Boyaval, P., Moineau, S., et al. (2007). CRISPR provides acquired resistance against viruses in prokaryotes. Science 315, 1709-1712. doi: 10.1126/science.1138140

Barrangou, R., and Horvath, P. (2017). A decade of discovery: CRISPR functions and applications. Nat. Microbiol. 2:17092. doi: 10.1038/nmicrobiol.2017.92

Barros, M. P., França, C. T., Lins, R. H., Santos, M. D., Silva, E. J., Oliveira, M. B., et al. (2014). Dynamics of CRISPR loci in microevolutionary process of Yersinia pestis strains. PLoS One 9:e108353. doi: 10.1371/journal.pone.0108353

Bikard, D., and Barrangou, R. (2017). Using CRISPR-Cas systems as antimicrobials. Curr. Opin. Microbiol. 37, 155-160. doi: 10.1016/j.mib.2017.08.005

Bikard, D., Hatoum-Aslan, A., Mucida, D., and Marraffini, L. A. (2012). CRISPR interference can prevent natural transformation and virulence acquisition during in vivo bacterial infection. Cell Host Microbe 12, 177-186. doi: 10.1016/ j.chom.2012.06.003

Boudry, P., Semenova, E., Monot, M., Datsenko, K. A., Lopatina, A., Sekulovic, O., et al. (2015). Function of the CRISPR-Cas system of the human pathogen Clostridium difficile. mBio 6:e01112-15. doi: 10.1128/mBio.01112-15

Bourgogne, A., Garsin, D. A., Qin, X., Singh, K. V., Sillanpaa, J., Yerrapragada, S., et al. (2008). Large scale variation in Enterococcus faecalis illustrated by the genome analysis of strain OG1RF. Genome Biol. 9:R110. doi: 10.1186/gb-20089-7-r110

Brouns, S. J., Jore, M. M., Lundgren, M., Westra, E. R., Slijkhuis, R. J., Snijders, A. P., et al. (2008). Small CRISPR RNAs guide antiviral defense in prokaryotes. Science 321, 960-964. doi: 10.1126/science.1159689 
Brown, S. D., Nagaraju, S., Utturkar, S., De Tissera, S., Segovia, S., Mitchell, W., et al. (2014). Comparison of single-molecule sequencing and hybrid approaches for finishing the genome of Clostridium autoethanogenum and analysis of CRISPR systems in industrial relevant Clostridia. Biotechnol. Biofuels 7:40. doi: 10.1186/ 1754-6834-7-40

Cartman, S. T., Kelly, M. L., Heeg, D., Heap, J. T., and Minton, N. P. (2012). Precise manipulation of the Clostridium difficile chromosome reveals a lack of association between the $t c d C$ genotype and toxin production. Appl. Environ. Microbiol. 78, 4683-4690. doi: 10.1128/AEM.00249-12

Charpentier, E., Richter, H., van der Oost, J., and White, M. F. (2015). Biogenesis pathways of RNA guides in archaeal and bacterial CRISPR-Cas adaptive immunity. FEMS Microbiol. Rev. 428-441. doi: 10.1093/femsre/fuv023

Cheng, F., Gong, L., Zhao, D., Yang, H., Zhou, J., Li, M., et al. (2017). Harnessing the native type I-B CRISPR-Cas for genome editing in a polyploid archaeon. J. Genet. Genomics 44, 541-548. doi: 10.1016/j.jgg.2017.09.010

Cohen, J. E., Wang, R., Shen, R.-F., Wu, W. W., and Keller, J. E. (2017). Comparative pathogenomics of Clostridium tetani. PLoS One 12:e0182909. doi: 10.1371/journal.pone.0182909

Cui, Y., Li, Y., Gorgé, O., Platonov, M. E., Yan, Y., Guo, Z., et al. (2008). Insight into microevolution of Yersinia pestis by clustered regularly interspaced short palindromic repeats. PLoS One 3:e2652. doi: 10.1371/journal.pone.0002652

Dapa, T., Leuzzi, R., Ng, Y. K., Baban, S. T., Adamo, R., Kuehne, S. A., et al. (2013). Multiple factors modulate biofilm formation by the anaerobic pathogen Clostridium difficile. J. Bacteriol. 195, 545-555. doi: 10.1128/JB.01980-1912

Garneau, J. E., Dupuis, M.-V., Villion, M., Romero, D. A., Barrangou, R., Boyaval, P., et al. (2010). The CRISPR/Cas bacterial immune system cleaves bacteriophage and plasmid DNA. Nature 468, 67-71. doi: 10.1038/nature 09523

Grissa, I., Vergnaud, G., and Pourcel, C. (2007). The CRISPRdb database and tools to display CRISPRs and to generate dictionaries of spacers and repeats. BMC Bioinformatics 8:172. doi: 10.1186/1471-2105-8-172

Hargreaves, K. R., and Clokie, M. R. J. (2014). Clostridium difficile phages: Still difficult? Front. Microbiol. 5:184. doi: 10.3389/fmicb.2014.00184

Hargreaves, K. R., Flores, C. O., Lawley, T. D., Clokie, R. J., Trevor, D., Hargreaves, K. R., et al. (2014). Abundant and diverse clustered regularly interspaced short palindromic repeat spacers in Clostridium difficile strains and prophages target multiple phage types within this pathogen. mBio 5:e01045-13. doi: 10.1128/ mBio.01045-13

Hochstrasser, M. L., Taylor, D. W., Kornfeld, J. E., Nogales, E., and Doudna, J. A. (2016). DNA targeting by a minimal CRISPR RNA-guided cascade. Mol. Cell 63, 840-851. doi: 10.1016/j.molcel.2016.07.027

Hong, W., Zhang, J., Cui, G., Wang, L., and Wang, Y. (2018). Multiplexed CRISPR-Cpfl-mediated genome editing in Clostridium difficile toward the understanding of pathogenesis of C. difficile infection. ACS Synth. Biol. 7, 1588-1600. doi: 10.1021/acssynbio.8b00087

Høyland-Kroghsbo, N. M., Paczkowski, J., Mukherjee, S., Broniewski, J., Westra, E., Bondy-Denomy, J., et al. (2016). Quorum sensing controls the Pseudomonas aeruginosa CRISPR-Cas adaptive immune system. Proc. Natl. Acad. Sci. U.S.A. 114, 131-135. doi: 10.1073/pnas.1617415113

Hsu, P. D., Lander, E. S., and Zhang, F. (2014). Development and applications of CRISPR-Cas9 for genome engineering. Cell 157, 1262-1278. doi: 10.1016/ J.CELL.2014.05.010

Jackson, S. A., McKenzie, R. E., Fagerlund, R. D., Kieper, S. N., Fineran, P. C., and Brouns, S. J. J. (2017). CRISPR-Cas: adapting to change. Science 356:eaal5056. doi: $10.1126 /$ science.aal5056

Kieper, S. N., Almendros, C., Behler, J., McKenzie, R. E., Nobrega, F. L., Haagsma, A. C., et al. (2018). Cas4 facilitates PAM-compatible spacer selection during CRISPR adaptation. Cell Rep. 22, 3377-3384. doi: 10.1016/J.CELREP.2018.02. 103

Kint, N., Janoir, C., Monot, M., Hoys, S., Soutourina, O., Dupuy, B., et al. (2017). The alternative sigma factor $\sigma \mathrm{B}$ plays a crucial role in adaptive strategies of Clostridium difficile during gut infection. Environ. Microbiol. 19, 1933-1958. doi: 10.1111/1462-2920.13696

Koonin, E. V., Makarova, K. S., and Zhang, F. (2017). Diversity, classification and evolution of CRISPR-Cas systems. Curr. Opin. Microbiol. 37, 67-78. doi: 10.1016/j.mib.2017.05.008

Koskenniemi, K., Laakso, K., Koponen, J., Kankainen, M., Greco, D., Auvinen, P., et al. (2011). roteomics and transcriptomics characterization of bile stress response in probiotic Lactobacillus rhamnosus GG. Mol. Cell. Proteomics 10, M110.002741. doi: 10.1074/mcp.M110.002741

Kuehne, S. A., Heap, J. T., Cooksley, C. M., Cartman, S. T., and Minton, N. P. (2011). ClosTron-mediated engineering of Clostridium. Methods Mol. Biol. 765, 389-407. doi: 10.1007/978-1-61779-197-0_23

Lee, H., Zhou, Y., Taylor, D. W., and Sashital, D. G. (2018). Cas4-dependent prespacer processing ensures high-fidelity programming of CRISPR arrays. Mol. Cell 70, 48.e5-59.e5. doi: 10.1016/j.molcel.2018.03.003

Louwen, R., Staals, R. H. J., Endtz, H. P., van Baarlen, P., and van der Oost, J. (2014). The role of CRISPR-Cas systems in virulence of pathogenic bacteria. Microbiol. Mol. Biol. Rev. 78, 74-88. doi: 10.1128/MMBR.00039-13

Maier, L.-K., Dyall-Smith, M., and Marchfelder, A. (2015). The adaptive immune system of Haloferax volcanii. Life 5, 521-537. doi: 10.3390/life5010521

Maier, L.-K., Lange, S. J., Stoll, B., Haas, K. A., Fischer, S. M., Fischer, E., et al. (2013a). Essential requirements for the detection and degradation of invaders by the Haloferax volcanii CRISPR/Cas system I-B. RNA Biol. 10, 865-874. doi: $10.4161 /$ rna.24282

Maier, L.-K., Stoll, B., Brendel, J., Fischer, S., Pfeiffer, F., Dyall-Smith, M., et al. (2013b). The ring of confidence: a haloarchaeal CRISPR/Cas system. Biochem. Soc. Trans. 41, 374-378. doi: 10.1042/BST20120263

Maikova, A., Peltier, J., Boudry, P., Hajnsdorf, E., Kint, N., Monot, M., et al. (2018). Discovery of new type I toxin-antitoxin systems adjacent to CRISPR arrays in Clostridium difficile. Nucleic Acids Res. 46, 4733-4751. doi: 10.1093/nar/gky124

Makarova, K. S., Haft, D. H., Barrangou, R., Brouns, S. J. J., Charpentier, E., Horvath, P., et al. (2011). Evolution and classification of the CRISPR-Cas systems. Nat. Rev. Microbiol. 9, 467-477. doi: 10.1038/nrmicro2577

Makarova, K. S., Wolf, Y. I., Alkhnbashi, O. S., Costa, F., Shah, S. A., Saunders, S. J., et al. (2015). An updated evolutionary classification of CRISPR-Cas systems. Nat. Rev. Microbiol. 13, 722-736. doi: 10.1038/nrmicro3569

Mandin, P., Repoila, F., Vergassola, M., Geissmann, T., and Cossart, P. (2007). Identification of new noncoding RNAs in Listeria monocytogenes and prediction of mRNA targets. Nucleic Acids Res. 35, 962-974. doi: 10.1093/nar/gkl1096

Marraffini, L. A. (2015). CRISPR-Cas immunity in prokaryotes. Nature 526, 55-61. doi: $10.1038 /$ nature15386

McAllister, K. N., Bouillaut, L., Kahn, J. N., Self, W. T., and Sorg, J. A. (2017). Using CRISPR-Cas9-mediated genome editing to generate C. difficile mutants defective in selenoproteins synthesis. Sci. Rep. 7:14672. doi: 10.1038/s41598017-15236-5

Melnikow, E., Schoenfeld, C., Spehr, V., Warrass, R., Gunkel, N., Duszenko, M., et al. (2008). A compendium of antibiotic-induced transcription profiles reveals broad regulation of Pasteurella multocida virulence genes. Vet. Microbiol. 131, 277-292. doi: 10.1016/j.vetmic.2008.03.007

Miller, M. B., and Bassler, B. L. (2001). Quorum sensing in bacteria. Annu. Rev. Microbiol. 55, 165-199. doi: 10.1146/annurev.micro.55.1.165

Mukhopadhyay, A., Redding, A. M., Joachimiak, M. P., Arkin, A. P., Borglin, S. E., Dehal, P. S., et al. (2007). Cell-wide responses to low-oxygen exposure in Desulfovibrio vulgaris Hildenborough. J. Bacteriol. 189, 5996-6010. doi: 10.1128/JB.00368-07

Nale, J. Y., Chutia, M., Carr, P., Hickenbotham, P. T., Clokie, M. R. J., and Abedon, S. T. (2016). ' Get in Early '; biofilm and Wax Moth (Galleria mellonella) Models reveal new insights into the therapeutic potential of Clostridium difficile bacteriophages. 7, 1-16. doi: 10.3389/fmicb.2016.01383

Negahdaripour, M., Nezafat, N., Hajighahramani, N., Rahmatabadi, S. S., and Ghasemi, Y. (2017). Investigating CRISPR-Cas systems in Clostridium botulinum via bioinformatics tools. Infect. Genet. Evol. 54, 355-373. doi: 10. 1016/J.MEEGID.2017.06.027

Nozawa, T., Furukawa, N., Aikawa, C., Watanabe, T., Haobam, B., Kurokawa, K., et al. (2011). CRISPR inhibition of prophage acquisition in Streptococcus pyogenes. PLoS One 6:e19543. doi: 10.1371/journal.pone.0019543

Nuñez, J. K., Lee, A. S. Y., Engelman, A., and Doudna, J. A. (2015). Integrasemediated spacer acquisition during CRISPR-Cas adaptive immunity. Nature 519, 193-198. doi: 10.1038/nature14237

Palmer, K. L., and Gilmore, M. S. (2010). Multidrug-resistant enterococci lack CRISPR-cas. mBio 1:e00227-10. doi: 10.1128/mBio.00227-10

Patterson, A. G., Jackson, S. A., Taylor, C., Evans, G. B., Salmond, G. P. C., Przybilski, R., et al. (2016). Quorum sensing controls adaptive immunity through the regulation of multiple CRISPR-Cas systems. Mol. Cell 64, 1102-1108. doi: 10.1016/j.molcel.2016.11.012 
Pearson, B. M., Louwen, R., van Baarlen, P., and van Vliet, A. H. M. (2015). Differential distribution of type II CRISPR-Cas systems in agricultural and nonagricultural Campylobacter coli and Campylobacter jejuni isolates correlates with lack of shared environments. Genome Biol. Evol. 7, 2663-2679. doi: 10.1093/gbe/evv174

Peng, L., Pei, J., Pang, H., Guo, Y., Lin, L., and Huang, R. (2014). Whole genome sequencing reveals a novel CRISPR system in industrial Clostridium acetobutylicum. J. Ind. Microbiol. Biotechnol. 41, 1677-1685. doi: 10.1007/ s10295-014-1507-3

Pettengill, J. B., Timme, R. E., Barrangou, R., Toro, M., Allard, M. W., Strain, E., et al. (2014). The evolutionary history and diagnostic utility of the CRISPR-Cas system within Salmonella enterica ssp. enterica. PeerJ 2:e340. doi: 10.7717/peerj. 340

Poehlein, A., Zverlov, V. V., Daniel, R., Schwarz, W. H., and Liebl, W. (2013). Complete genome sequence of Clostridium stercorarium subsp. stercorarium strain DSM 8532, a thermophilic degrader of plant cell wall fibers. Genome Announc. 1:e0007313. doi: 10.1128/genomeA.00073-13

Pyne, M. E., Bruder, M. R., Moo-Young, M., Chung, D. A., and Chou, C. P. (2016). Harnessing heterologous and endogenous CRISPR-Cas machineries for efficient markerless genome editing in Clostridium. Sci. Rep. 6:25666. doi: $10.1038 /$ srep25666

Ratner, H. K., Sampson, T. R., and Weiss, D. S. (2015). I can see CRISPR now, even when phage are gone. Curr. Opin. Infect. Dis. 28, 267-274. doi: 10.1097/QCO. 0000000000000154

Rodriguez, A. M., Callahan, J. E., Fawcett, P., Ge, X., Xu, P., and Kitten, T. (2011). Physiological and molecular characterization of genetic competence in Streptococcus sanguinis. Mol. Oral Microbiol. 26, 99-116. doi: 10.1111/j.20411014.2011.00606.x

Sekulovic, O., Garneau, J. R., Néron, A., and Fortier, L. C. (2014). Characterization of temperate phages infecting Clostridium difficile isolates of human and animal origins. Appl. Environ. Microbiol. 80, 2555-2563. doi: 10.1128/AEM.002 37-14

Semenova, E., Jore, M. M., Datsenko, K. A., Semenova, A., Westra, E. R., Wanner, B., et al. (2011). Interference by clustered regularly interspaced short palindromic repeat (CRISPR) RNA is governed by a seed sequence. Proc. Natl. Acad. Sci. U.S.A. 108, 10098-10103. doi: 10.1073/pnas.1104144108

Sesto, N., Touchon, M., Andrade, J. M., Kondo, J., Rocha, E. P. C., Arraiano, C. M., et al. (2014). A PNPase dependent CRISPR system in Listeria. PLoS Genet. 10:e1004065. doi: 10.1371/journal.pgen.1004065

Shah, S. A., Erdmann, S., Mojica, F. J. M., and Garrett, R. A. (2013). Protospacer recognition motifs. RNA Biol. 10, 891-899. doi: 10.4161/rna.23764

Shmakov, S. A., Sitnik, V., Makarova, K. S., Wolf, Y. I., Severinov, K. V., and Koonin, E. V. (2017). The CRISPR spacer space is dominated by sequences from species-specific mobilomes. mBio 8:e01397-17. doi: 10.1128/mBio.01397-17

Soavelomandroso, A. P., Gaudin, F., Hoys, S., Nicolas, V., Vedantam, G., Janoir, C., et al. (2017). Biofilm structures in a mono-associated mouse model of Clostridium difficile infection. Front. Microbiol. 8:2086. doi: 10.3389/fmicb. 2017.02086

Sorek, R., Kunin, V., and Hugenholtz, P. (2008). CRISPR - a widespread system that provides acquired resistance against phages in bacteria and archaea. Nat. Rev. Microbiol. 6, 181-186. doi: 10.1038/nrmicro1793
Sorek, R., Lawrence, C. M., and Wiedenheft, B. (2013). CRISPR-mediated adaptive immune systems in bacteria and archaea. Annu. Rev. Biochem. 82, 237-266. doi: 10.1146/annurev-biochem-072911-172315

Soutourina, O. A., Monot, M., Boudry, P., Saujet, L., Pichon, C., Sismeiro, O., et al. (2013). Genome-wide identification of regulatory RNAs in the human pathogen Clostridium difficile. PLoS Genet. 9:e1003493. doi: 10.1371/journal. pgen.1003493

Stachler, A.-E., and Marchfelder, A. (2016). Gene repression in Haloarchaea using the CRISPR (Clustered Regularly Interspaced Short Palindromic Repeats)-Cas I-B system. J. Biol. Chem. 291, 15226-15242. doi: 10.1074/jbc.M116.724062

Takeuchi, N., Wolf, Y. I., Makarova, K. S., and Koonin, E. V. (2012). Nature and intensity of selection pressure on CRISPR-associated genes. J. Bacteriol. 194, 1216-1225. doi: 10.1128/JB.06521-11

Timme, R. E., Pettengill, J. B., Allard, M. W., Strain, E., Barrangou, R., Wehnes, C., et al. (2013). Phylogenetic diversity of the enteric pathogen Salmonella enterica subsp. enterica inferred from genome-wide reference-free SNP characters. Genome Biol. Evol. 5, 2109-2123. doi: 10.1093/gbe/evt159

Wang, H., La Russa, M., and Qi, L. S. (2016). CRISPR/Cas9 in genome editing and beyond. Annu. Rev. Biochem. 85, 227-264. doi: 10.1146/annurev-biochem060815-014607

Wang, S., Hong, W., Dong, S., Zhang, Z.-T., Zhang, J., Wang, L., et al. (2018). Genome engineering of Clostridium difficile using the CRISPR-Cas9 system. Clin. Microbiol. Infect. doi: 10.1016/j.cmi.2018.03.026 [Epub ahead of print].

Westra, E. R., Buckling, A., and Fineran, P. C. (2014). CRISPR-Cas systems: beyond adaptive immunity. Nat. Rev. Microbiol. 12, 317-326. doi: 10.1038/nrmicro 3241

Wiedenheft, B., van Duijn, E., Bultema, J. B., Waghmare, S. P., Zhou, K., Barendregt, A., et al. (2011). RNA-guided complex from a bacterial immune system enhances target recognition through seed sequence interactions. Proc. Natl. Acad. Sci. U.S.A. 108, 10092-10097. doi: 10.1073/pnas.110271 6108

Zegans, M. E., Wagner, J. C., Cady, K. C., Murphy, D. M., Hammond, J. H., and O'Toole, G. A. (2009). Interaction between bacteriophage DMS3 and host CRISPR region inhibits group behaviors of Pseudomonas aeruginosa. J. Bacteriol. 191, 210-219. doi: 10.1128/JB.00797-08

Zhang, J., Zong, W., Hong, W., Zhang, Z.-T., and Wang, Y. (2018). Exploiting endogenous CRISPR-Cas system for multiplex genome editing in Clostridium tyrobutyricum and engineer the strain for high-level butanol production. Metab. Eng. 47, 49-59. doi: 10.1016/j.ymben.2018.03.007

Conflict of Interest Statement: The authors declare that the research was conducted in the absence of any commercial or financial relationships that could be construed as a potential conflict of interest.

Copyright (c) 2018 Maikova, Severinov and Soutourina. This is an open-access article distributed under the terms of the Creative Commons Attribution License (CC BY). The use, distribution or reproduction in other forums is permitted, provided the original author(s) and the copyright owner(s) are credited and that the original publication in this journal is cited, in accordance with accepted academic practice. No use, distribution or reproduction is permitted which does not comply with these terms. 